\title{
«L'eterno brivido del mistero». Il fantastico nella produzione novellistica di Grazia Deledda
}

\author{
Silvia Zangrandi ${ }^{1}$
}

Ricevuto: 19 gennaio 2018 / Modificato: 10 gennaio 2019 / Accettato: 28 giugno 2019

Riassunto. Nella produzione novellistica di Grazia Deledda si trovano evidenti tracce di fantastico. Si tratta di un fantastico che si muove tra strano e meraviglioso, tra rêverie (intesa come una situazione in cui l'ìo, dimentico della sua storia contingente, lascia errare il proprio spirito e gode così di una libertà simile a quella del sogno) e narrazioni di superstizioni e leggende popolari in cui si avvicendano maghi dai poteri sovrumani, statuette in cui sono conficcati spilloni, giaculatorie propiziatorie, uso di olio santo o paramenti sacerdotali per fare riti magici. A onor del vero, tutta la produzione deleddiana reca tracce di incantamenti e mistero; tuttavia il presente studio intende indagare, all'interno della produzione novellistica, solo un aspetto del fantastico deleddiano, quello rappresentato dalle figure fantasmatiche. Deledda pare trovare nella forma del racconto la formula più adatta a unire l'elemento magico a quello realistico rappresentato dal mondo barbaricino.

Parole chiave: Deledda, fantastico, novelle, esitazione, Todorov.

\section{[en] «The eternal shiver of mystery». The Fantastic in Grazia Deledda's short stories}

\begin{abstract}
The short stories of Grazia Deledda present evident traces of the fantastic. But it is a particular kind of fantastic, one which wavers between the strange and the marvelous, between the reverie (a situation in which the ego, forgetful of its contingent history, lets its spirit wander and thus enjoys a freedom similar to that of the dream) and tales of superstition and popular legends. A fantastic where we meet magicians with superhuman powers, propitiatory litanies, holy oil or priestly vestments used for magical rites. Traces of enchantment and mystery can actually be found in all Deledda's works. However, this study aims at investigating only one element of Deledda's fantastic: the ghostly figures. Deledda seems to find in the short story the most suitable formula, one which combines the magical world with the realistic one of Barbagia, where she lived.
\end{abstract}

Keywords: Deledda, Fantastic, Short stories, Hesitation, Todorov.

Come citare: Zangrandi, Silvia (2019): «"L'eterno brivido del mistero”. Il fantastico nella produzione novellistica di Grazia Deledda», Cuadernos de Filología Italiana, 26, pp. 233-243.

1 Università IULM. Dipartimento di Studi umanistici, via Carlo Bo 1, 20143 - Milano.

silvia.zangrandi@iulm.it 
Nella produzione novellistica di Grazia Deledda si trovano evidenti tracce di fantastico. Si tratta di un fantastico che si muove tra strano e meraviglioso, tra rêverie (intesa, per dirla con Bachelard (2008), come una situazione in cui l'ìo, dimentico della sua storia contingente, lascia errare il proprio spirito e gode così di una libertà simile a quella del sogno), tra narrazioni di superstizioni e leggende popolari in cui si avvicendano maghi dai poteri sovrumani, statuette in cui sono conficcati spilloni, giaculatorie propiziatorie, uso di olio santo o paramenti sacerdotali per fare riti magici. Deledda è convinta che «molte delle leggende sarde hanno un vero valore storico, specialmente quelle di talune chiese e di qualche montagna [...]. Formerebbero, se ben raccolte e ben studiate, degli elementi, dirò anzi dei documenti vivi, utili per la storia sarda» (Deledda 1999: 19) ${ }^{2}$. Nel discorso ufficiale per il conferimento del Nobel a Grazia Deledda, il critico svedese Henrik Schück (1966: 19)sottolineò la capacità della scrittrice di fondere natura e storia narrata.

Nell'arte di dipingere la natura sono ben pochi i letterati europei che possono starle a pari. Grazia Deledda non fa sfoggio di colori brillanti, ma la natura che essa descrive ha i contorni semplici e grandiosi del paesaggio antico. Tutto ne ha la casta pienezza e la maestà. È una natura animata in modo meraviglioso, che si armonizza perfettamente con la psicologia dei personaggi.

A onor del vero tutta la produzione deleddiana reca tracce di incantamenti e mistero anche quando la narrazione è realistica; solo due esempi tra i tanti: in Il pastorello (dalla raccolta Il dono di Natale) si legge: «forse i giganti esistono davvero nel misterioso mondo delle montagne; sono essi che accumulano le roccie e coltivano le quercie sempre rigogliose e fresche. Ma noi, abitanti delle città, non li vediamo perché essi si nascondono al nostro apparire» (Deledda 1996d: 227); in L'avventore (dalla raccolta La vigna sul mare), l'uomo sconosciuto venuto da chissà dove, dagli occhi azzurri, dal lungo viso malaticcio che porta con sé un'aria esotica, ambigua e misteriosa, offre alla povera fioraia del cibo che assume forza simbolica e diffonde un'atmosfera di prodigio nel bar.

Le storie che i vecchi pastori raccontavano furono per lei una ricca fonte di scoperte. Inoltre il paesaggio sardo «ha in se stesso qualcosa di meraviglioso e di leggendario [...] è seriamente immaginoso, pieno di superstizioni bizzarre e infinite» (Deledda 1999: 4). Se si accoglie la classificazione di Todorov, nel meraviglioso puro possiamo far confluire le numerose fiabe e i racconti di fate. Deledda racconta delle «domus de janas», i celebri monumenti megalitici, abitazioni o tombe preistoriche, che la fantasia popolare vuole ancora abitate da certe piccole fate, generose o malefiche a seconda dei casi (si legga ad esempio L'anellino d'argento dalla raccolta Il dono di Natale), da folletti, come Surtore che si nasconde nelle case, invisibile a tutti, e si diverte a nascondere gli oggetti. Nel racconto La grazia (dalla raccolta Sole d'estate) l'incontro, nel luogo roccioso dove si dice vivano le janas, con una

2 Sin da giovane Deledda mostra interesse per il folclore sardo; si propone così a Angelo De Gubernatis, docente presso l'Università di Roma e fondatore della Società italiana per il folclore, come collaboratrice per svolgere ricerche attorno ai costumi della sua regione. Gli studi deleddiani, in particolare dedicati alla zona del nuorese, confluiscono a puntate sulla Rivista delle tradizioni popolari italiane, diretta appunto da De Gubernatis, tra la fine del 1893 e il 1895 col titolo Tradizioni popolari di Nuoro in Sardegna. Il sodalizio che nasce tra i due incoraggiò Deledda a intraprendere la carriera di scrittrice: sull'argomento cfr. il carteggio tra Deledda e De Gubernatis (Masini 2007). Sugli studi deleddiani riguardo al folclore cfr. Delitala (1992). 
vecchia vestita di nero che ha una fiducia cieca nella «potenza magica della parola» (Deledda 1996e: 127), diventerà il viatico che accompagnerà la narratrice nel suo percorso letterario e le farà superare le critiche dei suoi compaesani ${ }^{3}$.

Il presente studio intende indagare, all'interno della produzione novellistica, solo un aspetto del fantastico deleddiano ${ }^{4}$ : quello rappresentato dalle figure fantasmatiche. In realtà, tracce di fantastico e ambientazioni inquietanti sono presenti anche nell'opera che l'ha resa famosa, Canne al vento. In apertura, il vecchio Efix, dopo una giornata di lavoro, al tramonto si mette in ascolto del «popolo della notte» fatto di presenze misteriose desunte da leggende e credenze dell'antico popolo sardo:

la luna saliva davanti a lui, e le voci della sera avvertivano l'uomo che la sua giornata era finita. Era il grido cadenzato del cuculo, il zirlio dei grilli precoci, qualche gemito d'uccello; era il sospiro delle canne e la voce sempre più chiara del fiume: ma era soprattutto un soffio, un ansito misterioso che pareva uscire dalla terra stessa: sì, la giornata dell'uomo lavoratore era finita, ma cominciava la vita fantastica dei folletti, delle fate, degli spiriti erranti. I fantasmi degli antichi baroni scendevano dalle rovine del castello sopra il paese di Galte [...]. Efix sentiva il rumore che le panas facevano nel lavar i loro panni giù al fiume, battendoli con uno stinco di morto, e credeva di intraveder l'ammattadore, il folletto con sette berretti entro i quali conserva un tesoro, balzar di qua e di là sotto il bosco di mandorli, inseguito dai vampiri con la coda di acciaio (Deledda 1913 [2013]: 28-29).

Tuttavia, Deledda pare trovare nella forma del racconto la formula più adatta a unire l'elemento magico a quello realistico ed elabora «una misura narrativa e stilistica adeguata alle peculiarità del mondo barbaricino attraverso una sua interpretazione in forme fantastiche» (Cerina 1996a: 7). Nella novella La chiesa nuova (dalla raccolta Sole d'estate) le immagini dei paesaggi romani si sovrappongono a quelle dell'isola lontana: la visione dei lecci millenari che fiancheggiano la strada di Roma trascolora nei boschi della sua infanzia e nella credenza che vuole che la Sardegna sia uno scoglio della leggendaria Atlantide. Come in un percorso fiabesco, all'uscita del bosco compare la chiesa nuova che, con «una metamorfosi fantastica» (Deledda 1996e: 120), diventa l'antica chiesetta della sua infanzia, ma «la gioia azzurra, l'illusione vestita d'iride» (Deledda 1996e: 119) dell'infanzia sfuma nelle immagini dolorose del presente: nella chiesa «c'era come un'allucinazione di luogo incantato [...], il pavimento mi parve azzurro come un vetro sul mare» (Deledda 1996e: 120).

Nell'alternarsi di realtà, come la richiesta angosciosa di un veleno per una dolce morte da parte di un malato, e di immaginazione, il paese favoloso «della sua stirpe antica» (Deledda 1996e: 120), la narratrice definisce il dolore «ospite inesorabile e divino [...], intermediario tra noi e Dio» (Deledda 1996e: 122). Attraverso la finzio-

3 Questa donna attribuisce alla capacità di scrivere della narratrice un potere magico, capace di arrivare al cuore della regina; per questa ragione la donna chiede alla narratrice di rivolgersi alla sovrana per porre fine all'ingiustizia subita da suo figlio. Riguardo ai racconti fiabeschi cfr. Mazzoni (2008).

4 Tzvetan Todorov per primo ha cercato di classificare il fantastico, fino a quel tempo per lo più relegato tra $i$ prodotti della letteratura di genere o di consumo. Todorov ha costruito una teoria della letteratura fantastica basata su alcune categorie. I racconti di Deledda di cui ci occuperemo oscillano tra lo strano puro, dove «on relate des événements qui peuvent parfaitement s'expliquer par les lois de la raison, mais qui sont [...] incroyables, extraordinaires [...] inquiétants» (Todorov 1970: 51), il fantastico strano dove «des événements qui paraissent surnaturels tout au long de l'histoire, y reçoivent à la fin une explication rationelle» (Todorov 1970: 49) e il fantastico puro, riscontrabile in testi che esitano tra spiegazione razionale e spiegazione soprannaturale. 
ne letteraria, alla ricerca di un significato da dare alla vita quando questa si avvia verso la fine, la narratrice attraversa una zona al limite tra presente e passato, tra la vita e il suo oltre in cui il paesaggio ricordato funge da sfondo irrinunciabile.

Anche il racconto Un grido nella notte (dalla raccolta Chiaroscuro) esplicita, con la sua apertura realistica, la necessità del fantastico deleddiano di poggiarsi sul dato geografico. Siamo a Nuoro, tre vecchi amici sono soliti passare la giornata seduti su una panchina di pietra vicina a una casetta; l'ambiente intorno a loro è descritto con precisione topografica: è il «vicinato di Sant'Ussula [...] allo sfolto della strada appare il monte Orthobene grigio e verde fra le due grandi ali azzurre dei monti d'Oliena e dei monti di Lula» (Deledda 1996b: 42). L'appartenenza alla Sardegna è la condizione indispensabile per Grazia Deledda per far rivivere leggende, miti e favole del suo mondo: «la trasformazione della Sardegna in macchina del fantastico è supportata [...] da dei macroscopici e scoperti simboli» (Marras 2010: 211). Come accade al fantastico novecentesco, poco dopo il racconto trasmigra nello straordinario: la giovane moglie di uno di loro, bella, forte, coraggiosa, una notte viene svegliata da un grido terribile. La donna, terrorizzata, si barrica in casa e il giorno seguente scopre vicino alla sua abitazione il cadavere di un giovane uomo. Da allora la donna prova profondi sensi di colpa per non aver prestato soccorso all'uomo; un giorno, durante la festa dei santi Cosimu e Damianu, si reca a pregare in una chiesetta, ma poco dopo ne esce sconvolta perché, mentre era all'interno, viene coinvolta in «un cerchio di persone che ballavano tenendosi per mano, senza canti, senza rumore $[\ldots]$ : erano i morti che ballavano [...], due mani magre e fredde strinsero la mia ... ed io dovetti ballare» (Deledda 1996b: 45). Deledda lungo la narrazione dà spiegazioni razionali - il delirio della donna, il suo senso di colpa, il rimpianto di non essere riuscita a soccorrere il giovane -; tuttavia la situazione descritta, benché surreale, non è totalmente improbabile grazie alle descrizioni dei luoghi ed è certamente inquietante. Con alta frequenza, l'inquietudine è data dalla presenza di esseri liminali, di misteriose apparizioni che non appartengono più al mondo dei vivi, ma con esso desiderano avere contatti.

In Lasciare o prendere (dalla raccolta Chiaroscuro) viene raccontata l'avventura di Giuseppe, un giovane uomo che la notte di Natale si lascia trascinare da altri giovani a bere. Durante la serata, però, si sente molto inquieto e ha l'impressione che qualcuno lo spinga verso casa: «una lieve vertigine gli velò la mente: figure conosciute eppure indistinte tornavano a circondarlo, come nella casa dove aveva passato la notte» (Deledda 1996b: 129). Tornato a casa, trova il cofanetto lasciato dalla vecchia serva contenente un tesoro del quale conosceva l'esistenza ma non sapeva dove si trovasse ${ }^{5}$. E ancora, protagonista di Il castello di Galtelli (dalla raccolta Leggende sarde) è l'ultimo barone che veglia sui ruderi del castello e sui tesori ivi nascosti. Costui, durante la notte, cammina nei dintorni mentre all'interno vivono altri spiriti. Questo spirito benevolo, una notte, chiede a un pastore di passaggio della

\footnotetext{
Alcuni racconti di Deledda risentono delle tendenze moraleggianti e pedagogiche dei racconti romantici di inizio secolo e si riallacciano alla forma narrativa minore del bozzetto della seconda metà dell'Ottocento (cfr. Fedi 1989). Enrico Cesaretti, in uno stimolante studio, dimostra che in alcune novelle è possibile trovare però anche tracce boccaccesche portatrici di una morale alternativa: la disobbedienza verso gli ordini ricevuti, spesso narrata dalla scrittrice, rivendica il desiderio di indipendenza del personaggio e la sua raggiunta maturità e segna il suo percorso di crescita (cfr. Cesaretti 2007). Nei personaggi deleddiani, in bilico tra desiderio di rispettare un ordine ed esigenza di trasgressione, si ritrova la stessa scrittrice che, nonostante le ingiunzioni a non continuare a scrivere, lascia la sua terra e si realizza artisticamente.
} 
legna per scaldare la sua casa. Costui, generosamente, gliela regala, ma, diventato stranamente ricco, tutti pensano che tale ricchezza derivi dal tesoro del barone. A questo proposito Deledda commenta: «la leggenda dei tesori ha così profonde radici da noi che non appena un individuo è riuscito, col suo lavoro e con la sua intelligenza, o magari con l'inganno e la perversità, ad acquistarsi qualche fortuna, subito la voce del popolino afferma che egli ha trovato un aschisorgiu, cioè un tesoro» (Deledda 1999: 4) ${ }^{6}$.

Anche la dama bianca (La dama bianca dalla raccolta Racconti sardi) è un fantasma che appare in sogno al pastore Bellia per indicargli dove si trova un magnifico tesoro. Il narratore è uno «strano tipo di sardo con una soave e bianca testa di santo [che racconta una storia] realmente avvenuta in questa terra delle leggende, delle storie cruente e soprannaturali, delle avventure inverosimili» (Deledda 1996a: 154). La donna, che risponde al nome di Donna Maria Croce $^{7}$, si presenta in sogno, vestita «con un mantello di velluto bianco che la copre da capo a piedi. Ha il volto bianco come il suo manto» (Deledda 1996a: 154); per tre notti di seguito visiterà Bellia e lo inciterà ad andare alla ricerca di un tesoro, fornendogli le coordinate esatte dove trovarlo ed esortandolo a fare presto. Ancora una volta, utilizzando il patrimonio culturale e il paesaggio sardo, ci viene descritto il luogo: «era un lembo bizzarro di paesaggio: campi deserti e selvaggi di montagna, picchi di roccie e di felci, circoscritti da boschi di elci secolari» (Deledda 1996a: 157). Secondo Natalino Sapegno, l'ambiente descritto «sembra emanare dalle figure e dalle situazioni come una sorta di alone fantastico, di luminosità diffusa» (Sapegno 1971 [1987]: xvii). Il giovane però, a causa di eventi imprevisti, arriverà tardi nel luogo indicato e troverà solo «un gran vaso di ferro arrugginito» (Deledda 1996a: 157). Molti enigmi sono sparsi lungo il racconto: l'insistenza sullo strano e l'ambiguo, sulla incredulità e il suo opposto provati dall'amico che ascolta la storia («nonostante la sua scettica ironia, egli sentì un brivido serpeggiargli per le reni», Deledda 1996a: 155), sull'indicazione di dati e luoghi precisi dove irrompe il fatto incredibile e infine la meraviglia e il terrore dei due giovani. Anche l'autenticazione di fatti inverosimili è un'ulteriore spia che contraddistingue la narrazione fantastica; infatti, viene trovato un testamento, che funge da oggetto mediatore ${ }^{8}$, che nomina Bellia erede del tesoro, tesoro che però, come sappiamo, non verrà trovato perché cercato in ritardo e, come scrive Deledda, «il

6 Intorno a questo castello si narra un altro episodio molto più intrigante: una donna del villaggio viene chiamata in aiuto e condotta al castello dove, dopo aver visto ogni genere di cose favolose e attraversato sale splendide, viene condotta in una camera dove una donna sta per partorire. Col suo aiuto la donna dà alla luce un bel bambino, ma le dice che il bimbo non è di questo mondo. Una simile storia viene narrata in Canne al vento dall'usuraia Kallina che ricorda l'incontro avvenuto vicino al castello con «un signore vestito dei colori del sole e dei monti» (Deledda 1913 [2013: 110]) che la prega di andare a chiamare la «Maestra di parto» e le dà alcune monete come ricompensa.

7 La donna si chiama Donna Maria Croce ma firmerà il testamento, scritto in logudorese, con il nome Maria Rughe; del cognome appare solo l'iniziale $\mathrm{M}^{* * *}$ a indicare che della nobildonna si possono raccontare i trascorsi ma non la sua completa identità. Benché abbreviato, la presenza del cognome attesta una certa autenticità del narrato e si fa illusione del vero. Per approfondimenti sul rapporto tra i nomi e il fantastico cfr. Zangrandi (2017).

8 A volte, a prova della verità dei fatti, c’è la presenza, per dirla con Lugnani, di un «oggetto mediatore [che] svolge la duplice funzione di prova della verità e di restauratore dell'ordine: ritrovato e ricollocato al suo posto, esso sana le lacerazioni o le distorsioni della realtà [...]; questi oggetti privilegiati stanno a testimoniare l'esistenza e la raggiungibilità [...] di piani di realtà diversi rispetto al quotidiano e al realistico» (Lugnani 1983: 187-189). 
diavolo [...] se alla fine di un certo tempo gli uomini non ritrovano il tesoro, se ne appropria lui stesso e se lo porta all'inferno» (Deledda 1999: 4).

L'oggetto mediatore interviene con frequenza nel fantastico novecentesco e anche in quello deleddiano: nel racconto Cose che si raccontano (dalla raccolta $L a$ casa del poeta) un vecchio cameriere a servizio in una clinica, dopo aver portato un brodo a una giovane donna morente che lo guarda in modo strano, la sogna di notte elegantemente vestita mentre gli chiede un bacio. Al suo rifiuto, ella con una spilla gli tatua sul braccio la lettera B. Al risveglio, l'uomo scopre che ha un tatuaggio sul braccio e che la donna è morta. Un vecchio scrittore che ascolta l'avventura dell'uomo spiega che è stata la sua pietà per la donna a procurargli il sogno e che l'anima della defunta era venuta a salutarlo. Restano però il tatuaggio che genera una spaccatura tra realtà e irrealtà, autentico oggetto mediatore che mette in comunicazione il mondo reale con quello fantastico e crea meraviglia, incredulità, «resistenza dell'ordine costituito al possibile disordine indotto o da un nuovo ordine che si fa strada o dall'inatteso riaffacciarsi di un ordine antico che si credeva superato» (Lugnani 1983: 195-196).

Le presenze fantasmatiche nei racconti di Deledda si giustificano con le parole della stessa scrittrice, che afferma in Lo spirito dentro la capanna (dalla raccolta $\mathrm{Il}$ sigillo d'amore): «questi spiriti, che abitano facilmente in molti posti, anche nei palazzi delle città e persino nei grandi alberghi, non mi riescono antipatici: quelli della capanna, poi, li ringraziavo di tenermi in quel luogo libero e pulito» (Deledda 1996c: 398). Il racconto narra di «un lieve strido, o meglio come un vagito lamentoso di un bambino appena nato» (Deledda 1996c: 397) uscire dalla porta quasi invisibile di una capanna in mezzo a un bosco. In quella capanna era avvenuto un delitto: un uomo, convinto di aver ucciso suo fratello, si era tolto la vita dalla disperazione, perciò tutti credono che dentro sia rimasto lo spirito inquieto dell'uomo. Il lettore, poco più avanti, viene informato dell'accaduto attraverso il racconto di una donna, la moglie del suicida, che si avvicina alla porta della capanna con un mazzolino di fiori in mano per acquietare il tormento del fantasma. In questo racconto, come altrove, a stemperare l'inquietudine creata dal racconto, giungono le parole della narratrice che tentano di dare una spiegazione razionale agli eventi: qui dice che il gemito proviene forse da un ramo di pino spezzato dal vento; in La dama bianca il personaggio, «nonostante la sua scettica ironia, [...] sentì un brivido serpeggiargli per le reni»; in Un grido nella notte giustifica le visioni della donna tramite il suo senso di colpa per non aver aiutato l'uomo morente. Insomma, pur narrando superstizioni, apparizioni di fate, fenomeni misteriosi, Deledda si mostra scettica e cerca sempre il modo di dare una spiegazione razionale. Del resto Todorov aveva avvertito che l'esitazione è il momento cruciale della narrativa fantastica: il fantastico è per lo studioso bulgaro «l'hésitation éprouvée par un être qui ne connaît que les lois naturelles, face à un événement en apparence surnaturel» (Todorov 1970: 29) e si verifica ogni volta che un fatto non può essere spiegato con le leggi del mondo a noi noto:

Ou bien il s'agit d'une illusion des sens, d'un produit de l'imagination et les lois du monde restent alors ce qu'elles sont ; ou bien l'événement a véritablement eu lieu, il est partie intégrante de la réalité, mais alors cette réalité est régiée par des lois inconnues de nous [...]. Le fantastique occupe le temps de cette incertitude (Todorov 1970: 29). 
Il racconto Il mago (dalla raccolta Racconti sardi) conferma l'affermazione todoroviana. In esso si narra di un giovane che, non riuscendo a diventare padre, si rivolge a un mago, convinto dalla moglie di essere oggetto di una fattura. Il mago lo invita in un luogo solitario, pieno di pietre, munito di una tovaglia, due candele e un fucile caricato a salve. In questo luogo, «reso fantastico dal chiarore croceo della luna al tramonto» (Deledda 1996a: 137), il sedicente mago recita parole magiche rivolgendosi a una pietra: la pietra risponde con parole incomprensibili, i ceri si spengono e l'uomo, come gli era stato chiesto dal mago, spara perché lo sparo del fucile segnalava la riuscita del rito magico. Inspiegabilmente però, il colpo, benché a salve, uccise il mago. Mesi dopo nacque un bambino: «sempre incredulo in fatto di magie, il forte pastore dai grandi occhi ardenti, non seppe mai spiegarsi come la pietra avesse parlato, come i ceri eransi spenti e come il fucile aveva fatto fuoco» (Deledda 1996a: 139); ebbe però la gioia di diventare padre e, in suffragio dell'anima del mago, fece celebrare una messa.

Anche nel racconto Lo spirito della madre (dalla raccolta Sole d'estate) emerge lo scetticismo della scrittrice nei confronti del supernaturale, qui rappresentato dalla protagonista che, involontariamente presente a una seduta spiritica, «capiva che si giocava agli spiriti $[\ldots]$; non ci credeva, lei, no [...] eppure un certo brivido le tremò fra scapola e scapola» (Deledda 1996e: 36). Qui si racconta che la giovane Lula si sente responsabile della morte della madre, scomparsa dopo che lei era fuggita di casa col figlio del padrone. Un giorno, mentre sta facendo le inalazioni per curare la gola, assiste a una seduta spiritica. Improvvisamente «si sentì come sollevata da una mano formidabile che la tirò su, in piedi, rigida e folle. Con una voce strana, che neppur lei riconosceva per sua» (Deledda 1996e: 36), disse alla medium di chiamare la mamma e lo spirito entrò in lei, pacificandola. La venuta del fantasma ha scopo rasserenante e non bellicoso. L'apertura, in cui si respira un'atmosfera di inquietudine data sia dal malessere che prova Lula, sia dalla nebbia creata dagli apparecchi per le inalazioni che dà alla gente l'aspetto di fantasmi e nasconde cose e persone presenti nell'ambiente, si risolve in un finale confortante. Infatti, la prima parte è incentrata sullo stato d'animo della ragazza addolorata per la morte della madre di cui si colpevolizza e sull'ambiente concreto di una sala per le inalazioni; in questa quotidianità si innesta la dimensione irreale causata dalla credenza popolare che vuole che, tramite un medium, si possano evocare gli spiriti. Lula si convince che «lo spirito della madre era in quel momento penetrato in lei» (Deledda 1996e: 37). L'aspetto fantastico e la dimensione sovrannaturale si esplicitano attraverso il turbamento di Lula che dapprima si sente sollevare e poi parla con voce strana. Il turbamento, però, si evolve in rasserenamento poiché lo spirito evocato sembra averla raggiunta, pacificata e finalmente liberata dalla sua inquietudine.

Alcuni racconti rispecchiano i principi espressi da Roger Caillois (1984: 35-36): «al fantastico occorre qualcosa d'involontario, di subito, un interrogativo inquieto, non meno che inquietante scaturito all'improvviso da chissà quali tenebre e che il suo stesso autore è stato costretto a prendere così come è venuto, a cui a volte ha desiderato perdutamente poter dare una risposta». In L'usuraio (dalla raccolta Il fanciullo nascosto) Deledda racconta le vicende di un usuraio morente. Questi ha accanto a sé un prete per un'ultima confessione quando, improvvisamente, gli sportelli dell'armadio della camera in cui si trova si aprono e, nonostante si tenti di chiuderli, subito si riaprono e «l'odore della canfora uscì come da una porta aperta sul giardino del morti» (Deledda 1996b: 268). È evidente che l'armadio rappresenta la porta che 
comunica con l'aldilà. «Dentro era apparsa come un fantasma una donna che dava le spalle alla stanza [...] Alessandra Madau quale la si vedeva nei giorni di festa» (Deledda 1996b: 267). Si tratta della vecchia padrona della casa che aveva rifiutato di sposare l'usuraio, nonostante fosse sul lastrico, poiché lei era nobile mentre lui era un semplice mercante: era stato l'uomo che, con i suoi prestiti, l'aveva ridotta in miseria. Una volta morta, la donna aveva iniziato a tormentarlo. L'usuraio, agonizzante, bacia il vestito di lei e finalmente si sente in pace. Il racconto si conclude però con l'impossibilità da parte del prete di chiudere l'armadio.

Apparizioni misteriose si susseguono nelle pagine deleddiane: è il caso della figura rossa di donna che porta nella sua cassetta «pizzi, ricami, merletti, tovaglie, scialli $[. .$.$] e un tappeto per tavola [...] con la frangia che pareva fatta con bionde$ ciglia di sirene» (Deledda 1996e: 113) raccontata in Il tappeto (dalla raccolta Sole d'estate). La donna ha «la mansuetudine veloce del cammello e la sveltezza rapace della zingara [...], pareva venisse dall'antica città, con un tesoro rubato a qualche principessa bizantina» (Deledda 1996e: 113). Tutto concorre a creare un'aura incantata, grazie soprattutto al tappeto, che aveva tinte cangianti difficili da descrivere perché

su un fondo carnicino dorato, degno della grande rosa centrale, si svolgevano corone di ghiande verdi, di foglie azzurre, di perle ovali rosse, di ghirigori d'oro [...] cuori che riassumevano tutti gli altri colori; e più fulgidi e vivi di quelli che si vedono sulle pareti di certe cappelle miracolose [...] alcuni verdi e azzurri [...] altri d'un viola livido come cuori di donne assassinate per amore (Deledda 1996e: 114).

Di fronte a questa fantasmagoria magica di colori ${ }^{9}$, che qui viene definita «cisterna d'incantesimo» (Deledda 1996e: 114), la donna si impossessa del tappeto e lo porta via con sé. «Il tappeto ha il valore di un'illusione con i suoi colori e i suoi simboli, portatori di significati che lei sola riesce a decifrare» (Cerina 1996b: 9). Anche Lo stracciaiolo del bosco (dalla raccolta Sole d'estate), arrivato da non si sa dove, reca con sé un mistero che si riverbera nei «vividi occhi verdognoli che parevano di diaspro» (Deledda 1996e: 107) e nel suo sacco misterioso dal quale emerge ogni tipo di mercanzia e dà «un'aria di spavento [...] una certa sfumatura di inquietudine fiabesca s'è diffusa nel cuore degli astanti, e anche nell'atmosfera intorno» (Deledda 1996e: 107-108). Quando il suo carico si sparpaglia sulla tavola, «l'involto e le scatole prendono subito un altro colore» (Deledda 1996e: 108), la magia investe gli oggetti e la straordinarietà dell'evento procura gioia in chi assiste all'evento e curiosità verso l'uomo sconosciuto e misterioso. I gesti sapienti dell'uomo, che estrae con cautela vassoi e candelabri, aumentano lo stupore, incentivato anche dall'ultimo chiarore del tramonto che dà loro «un'illusione di altare» (Deledda 1996e: 109).

Come si diceva in apertura, Deledda è molto interessata alle abitudini e al folclore sardo e ne fa spesso oggetto dei suoi scritti. In La festa del Cristo (dalla raccolta Chiaroscuro) un'atmosfera inquietante avvolge il racconto del pellegrinaggio. La folla che in processione si avvia verso il santuario viene descritta giocando sui colo-

9 È palese «la tendenza a trasferire in notazioni di puro colore uno stato d'animo, una situazione di sentimento» (Frattini 1974: 324). 
ri: le donne hanno le bende gialle sugli occhi, i giovani hanno abiti di velluto color oliva e di pelle gialla, i vecchi hanno riccioli e lunghissimi baffi e il cappuccio sul capo. Questi colori però contrastano con le falde del monte verdastro e con il prete Filia che apre il corteo; egli è «nero, nero e scarno [...] montava un cavallo nero con una stella bianca in fronte» (Deledda 1996b: 148). Irrompe improvvisamente un cavallo rosso indomito, cavalcato da un giovane «con la barba rossiccia come la criniera del suo cavallo» (Deledda 1996b: 149). Il cavaliere sembra selvaggio come il suo animale che corre e scalpita portando scompiglio tra la folla ordinata che cammina verso la chiesa. Questo puledro rosso, ${ }^{10}$ «bello come l'oro», compare minaccioso, preludendo a un improvviso cambiamento del tempo:

verso il tramonto il tempo si fece orribile. Era ai primi di maggio, ma sembrò si ritornasse nel cuore dell'inverno. Soffiava il vento di tramontana e tutti i monti [...] parvero sciogliersi in nuvole color di pietra. Se il sole riusciva un momento a brillare simile a una brage in mezzo alla cenere [...] la pioggia scrosciava sul paese, il vento ululava (Deledda 1996b: 150).

$\mathrm{Al}$ riparo della pioggia in una casa, il giovane informa che lo spirito del vecchio avaro presso il quale ha comprato il cavallo «non è stato accolto né in cielo né in terra e s'è rifugiato nel corpo dell'animale» (Deledda 1996e: 151) e continua a scalpitare come se volesse spezzare le pietre. Un'atmosfera fosca avvolge l'ambiente: la luna corre tra le nuvole, una donna nera passa nella strada «con un tizzone acceso per allontanare i cani che durante la notte possono essere diavoli o anime erranti» (Deledda 1996e: 152$)^{11}$. Il prete Filia è perseguitato da incubi e recita una giaculatoria ostinata e reitera «Cristo, Dio, aiutaci!»; due fratelli si scontrano crudelmente, brandendo coltelli. Il paesaggio partecipa a questa aura tragica: «la stessa erba tremava di freddo, i rialzi di terreno coperti di puleggio davan l'idea di cadaveri violacei in decomposizione stesi lungo la strada, nel crepuscolo livido [...]; il lieto pellegrinaggio andava, andava, attraverso i salti e le tancas, e pareva cambiato in mortorio» (Deledda 1996b: 153). Anche il Cristo «inspirava un certo terrore, così grande e pallido com'era nel chiarore dei ceri, sopra l'antico altare» (Deledda 1996b: 158). Infine, assistiamo alla morte di un bambino colpito dal cavallo rosso e al malore del prete Filia.

D'accordo con Spinazzola, Deledda potenzia le «risorse d'una fantasia nativa, applicata a trasfigurare leggendariamente una materia di vita quotidiana, vissuta fra realtà e surrealtà» (Spinazzola 1981 [1990: 12]). Il suo continuo bilanciarsi tra mondo reale e fantastico è ben visibile in Nel regno della pietra (dalla raccolta Le tentazioni) dove gli avvenimenti si alternano tra realtà geografica e un altrove misterioso e surreale. Il paesaggio rispecchia i fatti cruenti che accadono. L'ovile di Sidru Addas

10 Nel dizionario dei simboli, alla voce "cavallo" si legge: «una credenza, che sembra radicata nella memoria di tutti i popoli, associa in origine il cavallo alle tenebre del mondo ctonio, sia che emerga galoppando dalle viscere della terra, o che scaturisca dagli abissi del mare [...]. I cavalli della morte, o presagi di morte, abbondano dall'antichità greca al medioevo e sono diffusi in tutto il folklore europeo» (Chevalier, Gheerbant 1986 [2005: 223-227]). Anche nell'Apocalisse di san Giovanni il cavallo rosso e il suo cavaliere hanno il potere di togliere la pace dalla terra e di far sì che gli uomini si uccidano a vicenda.

11 «In tutte le mitologie il cane [...] è sempre stato associato alla morte, agli inferi, al mondo sotterraneo, ai regni invisibili governati dalle divinità ctonie [...]. La prima funzione mitica del cane, universalmente documentata, è quella di psicopompo: è la guida dell'uomo nella notte della morte, dopo esser stato il suo compagno nel giorno della vita» (Chevalier / Gheerbant 1986 [2005: 185]). 
si trova «in una falda di Monte Bacchitta, sotto una corona di mostruose roccie granitiche che lo difendevano dai venti freddi del Nord [...]; quando il vento taceva, un silenzio indescrivibile era lassù, sotto quelle mostruose roccie allineate, grigie, enigmatiche» (Deledda 1996a: 364). Ancora una volta «la scrittrice ritma paesisticamente il mistero cosmico da cui le creature discendono e con cui aspirano a ricongiungersi, nella pace dell'essere. La natura è un sacro spettacolo che 1'uomo contempla con animo commosso» (Spinazzola 1974: 110). Anche il cane «posato sulle zampe come un idolo mostruoso, con gli occhi rossi, fissi lontano, animava la selvaggia solitudine dell'ovile» (Deledda 1996a: 365); il volto dello Zio Sidru, poi, «pareva scolpito sulla pietra [...] pareva che la natura della pietra, fra cui viveva, si fosse identificata in lui» (Deledda 1996a: 365). Tutta la rabbia di Zio Sidru dopo la scoperta del furto del suo bestiame si trasferisce nel paesaggio: «era una notte di vento [...] poi la luna tramontò, le nuvole si fecero nere [...] di lontano udì il cane abbaiare furiosamente destando echi cavernosi. Pareva la voce di un demonio incatenato fra le roccie [...], il vento gli sferzava le spalle, il fianco, i capelli [...], solo gli urli rauchi e rabbiosi del cane, animavano la buia solitudine» (Deledda 1996a: 374).

Un'atmosfera misteriosa e drammatica avvolge il racconto e la presenza dell'antica reliquia posta sulla porta della stalla sembra essere un oggetto apotropaico e ogni volta che il pastore le passa accanto si fa tre segni di croce, convinto che la sua presenza proteggesse la mandria. Questa reliquia fatta di legno rosso, frammento del sangue coagulato di San Giorgio, crea un'aura magica: «il sincretismo pagano-cristiano di sostrato culturale permea la pagina deleddiana rafforzando la valenza magica in cui il gesto palese appartiene alla religione cristiana, ma quello sotterraneo, viscerale, al contatto primitivo e magico con le forze oscure della natura» (De Giovanni 1993: 24). D'accordo con Nicolò Mineo, «ancora una volta la risposta alla crisi di inizio secolo è cercata in un mondo e in un tempo, certamente mitizzati, visti come garanzia di armonizzazione in consolidate regole sociali e in un recuperato accordo uomo/natura» (Mineo 2010: 96). La sensibilità descrittiva di Grazia Deledda, il legame verso i luoghi, la natura, i costumi sardi, i bozzetti paesaggistici da lei creati, il materiale etnografico: tutto è integrato in una narrativa dai toni stregati, in un'atmosfera che intreccia l'elemento fantastico e magico con quello mimetico e realistico, dando vita a una produzione di forte impatto emotivo.

\section{Riferimenti bibliografici}

Bachelard, Gaston (1972[2008]): La poetica della rêverie, Bari, Edizione Dedalo.

Caillois, Roger (1984): Nel cuore del fantastico, postfazione di Guido Almansi, Milano, Feltrinelli.

Cerina, Giovanna (1996a): «Prefazione», in G. Deledda, Novelle: Nell'azzurro. Racconti sardi. L'ospite. Le tentazioni, Nuoro, Ilisso, vol. I, pp. 7-20.

Cerina, Giovanna (1996b): «Prefazione», in G. Deledda, Novelle: Sole d'estate. Il cedro del Libano, Nuoro, Ilisso, vol. VI, pp. 7-20.

Cesaretti, Enrico (2007): «“Oh, la disobbedienza...!” Meditazioni su una novella giovanile di Grazia Deledda», Italica, LXXXIV(4), pp. 723-734.

Chevalier, Jean / Gheerbant, Alain (1986[2005]): Dizionario dei simboli, Milano, Rizzoli.

De Giovanni, Neeria (1993): Come leggere Canne al vento di Grazia Deledda, Milano, Mursia. 
Deledda, Grazia (1996a): Novelle: Nell'azzurro. Racconti sardi. L'ospite. Le tentazioni, vol. I, Nuoro, Ilisso.

Deledda, Grazia (1996b): Novelle: Chiaroscuro. Il fanciullo nascosto, vol. III, Nuoro, Ilisso.

Deledda, Grazia (1996c): Novelle: Il ritorno del figlio. La bambina rubata. Il flauto nel bosco. Il sigillo d'amore, vol. IV, Nuoro, Ilisso.

Deledda, Grazia (1996d): Novelle: La casa del poeta. Il dono di Natale. La vigna sul mare, vol. V Nuoro, Ilisso.

Deledda, Grazia (1996e): Novelle: Sole d'estate. Il cedro del Libano, vol. VI, Nuoro, Ilisso.

Deledda, Grazia (1999): «Premessa», Leggende sarde, a cura di Dolores Turchi, Roma, Newton Compton.

Deledda, Grazia (1913[2013]): Canne al vento, Milano, Rizzoli.

Delitala, Enrica (1992): «Grazia Deledda e la Rivista delle tradizioni popolari italiane», in U. Collu (a c. di), Grazia Deledda nella cultura contemporanea. Atti del Seminario di Studi “Grazia Deledda e la cultura sarda fra ' 800 e '900, 2 voll., Nuoro, Consorzio per la Pubblica Lettura “S. Satta”, vol. I, pp. 307-312.

Fedi, Roberto (1989): «Bozzetto e racconto nel secondo Ottocento. La novella italiana», in Atti del Convegno di Caprarola 19-24 settembre 1988, Roma, Salerno, tomo I, pp. 587606.

Frattini, Alberto (1974): «Tecnica dell'aggettivazione cromatica in alcune novelle della Deledda», in Convegno nazionale di studi deleddiani (Nuoro 30 settembre 1972), Cagliari, Edizione Sarda Fossataro, pp. 321-332.

Lugnani, Lucio (1983): «Verità e disordine: il dispositivo dell'oggetto mediatore», in R. Ceserani et al. (a c. di), La narrazione fantastica. Le radici storiche di un modo narrativo, Pisa, Nistri-Lischi, pp. 177-287.

Masini, Roberta (2007): Lettere ad Angelo De Gubernatis (1892-1909), Cagliari, Centro di Studi Filologici Sardi / Cuec.

Marras, Margherita (2010): «Eredità e modernità di G. Deledda», in G. Pirodda (a c. di), Dalla quercia del monte al cedro del Libano. Le novelle di Grazia Deledda, Nuoro, ISRE Aipsa Edizioni, pp. 205-216.

Mazzoni, Cristina (2008): "“The loving re-education of a soul": Learning from fairy tales through Grazia Deledda and Cristina Campo», Quaderni d'Italianistica, XXIX(2), pp. 93-109.

Mineo, Nicolò (2010): «Le novelle del primo decennio del Novecento», in G. Pirodda (a c. di), Dalla quercia del monte al cedro del Libano. Atti del Convegno nazionale di studi 8-10 novembre 2007, Cagliari, ISRE Edizioni / AIPSA Edizioni, pp. 85-96.

Sapegno, Natalino ( $\left.{ }^{2} 1987\right)$ : «Prefazione», Romanzi e novelle, Milano, Mondadori, pp. xixxiii.

Schück, Henrik (1996): «Discorso ufficiale per il conferimento del premio Nobel a Grazia Deledda», in Grazia Deledda: premio Nobel per la letteratura 1926, Milano, Fratelli Fabbri, pp. 15-22.

Spinazzola, Vittorio (1974): «Grazia Deledda e il pubblico», in Atti del Convegno nazionale di studi deleddiani (Nuoro 30 settembre 1972), Cagliari, Editrice Sarda Fossataro, pp. 105-126.

Spinazzola, Vittorio ( $\left.{ }^{2} 1990\right)$ : «Prefazione», in Grazia Deledda, Romanzi sardi, Milano, Mondadori, pp. 11-43.

Todorov, Tzvetan (1970): Introduction à la littérature fantastique, Paris, Editions du Seuil.

Zangrandi, Silvia (2017): Fanta-onomastica. Scorribande onomastiche nella letteratura fantastica del Novecento, Pisa, ETS. 